\title{
CEPO-based Research on Evacuation Route for High-rise Building Fire
}

\author{
Mingkong Zhang ${ }^{1}$, Xiaobing $\mathrm{Hu}^{1,2}$ \\ ${ }^{1}$ Academy of Disaster Reduction and Emergency Management, Faculty of Geographical science, \\ Beijing Normal University, Beijing 100875, China \\ ${ }^{2}$ College of Electronic Information and Automation, Civil Aviation University of China, Tianjin, \\ 300300, China
}

\section{基于 CEPO 方法的高层建筑火灾疏散路径研究}

\author{
张明空 ${ }^{1}$, 胡小兵 ${ }^{1,2}$ \\ ${ }^{1}$ 北京师范大学, 地理科学学部, 减灾与应急管理研究院, 北京, 100875 , 中国 \\ 2 中国民航大学, 电子信息与自动化学院, 天津, 300300, 中国
}

\begin{abstract}
The decision-making problem of evacuation route in high-rise building fire is actually the dynamic path optimization problem under fire spreading. Since DPO method can not guarantee the actual optimal path, increase unnecessary evacuation route and extend evacuation time under fire, improving ripple spreading algorithm to increase the number of successful evacuation in the safe evacuation time and achieve $\mathrm{Co}$ evolutionary path optimization (CEPO), synchronizing the process of personal evacuation with the process of fire diffusion. Simulate evacuation process of high-rise building by fire dynamics simulator and evaluate the efficiency of DPO method and CEPO method. The result shows that the CEPO method can solve the above DPO problems through an off-line optimization, and greatly improve the efficiency of fire evacuation.
\end{abstract}

Keywords: Co-evolutionary path optimization; Evacuation route; High-rise building fire
摘要

高层建筑火灾疏散路径的决策问题实 际是火灾不断曼延情况下的动态路径优化 (Dynamic Path Optimization, DPO) 问题。为 了提高在安全疏散时间内成功疏散的人 数, 针对 DPO 方法无法保证实际最优路 径、疏散路径绕远、疏散时间延长的问 题, 改进涟渏扩散算法 (Ripple Spreading Algorithm, RSA), 在火灾情景下采用协同 进化路径优化方法 (Co-evolutionary path optimization, CEPO), 同步人员疏散过程与 火灾扩散过程。通过借助火灾扩散的动力 学模型, 对高层火灾疏散过程进行仿真, 评估 DPO 方法和 CEPO 方法的疏散效率。 结果表明 CEPO 方法通过一次离线优化就 可以解决上述 DPO 存在的问题, 大大提高 了火灾疏散效率。

关键词: 协同路径优化; 涟渏扩散算法; 疏散路径; 高层建筑火灾

\section{1. 引言}

城市高层建筑数量与日俱增的同时, 也带来了许多社会安全问题, 尤其是高层 火灾。在各类火灾中, 高层建筑的火灾对

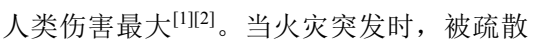
人员往往由于对火源情况认识不清、对环 
境陌生而难以选择有效逃生路径 ${ }^{[3]}$, 因此极 易闯入火灾区域而造成大量人员伤亡。

高层建筑火灾下应急疏散路径的求解 问题属于动态路径优化 (Dynamic Path Optimization, DPO) 问题。由于火灾的发展 是随时间动态变化的, 因此疏散路径要根 据火灾态势实时进行动态调整。传统算法 的求解思路一般都是基于实时灾害情况进 行在线优化, 一旦原始路网发生变化, 疏 散路径就需要在线重新计算, 其实, 每次 疏散路径的计算都是基于当前火灾情况的 静态路径优化算法 (Static Path Optimization, SPO)。目前解决这一问题的 算法有很多, 如 Dijkstra 算法、Floyd 算 法、蚁群算法、A*算法等, 特别是蚁群算法 作为启发式算法具有适用性强、易于同其 他算法结合等优点, 得到了广泛应用 [4][6]。为了提高动态路径优化算法的计算效 率, 许多路径优化算法的研究多从改进算 法本身为出发点 ${ }^{[7]-[9]}$, 从而局限于一些参数 配置的优化而忽略了真实环境下外部环境 随时间的动态改变, 例如: 为了提高在线 计算时间的速度, 文献[10]-[13]改进了每次 路网发生变化时求解最新疏散路径的速 度。即使如此 DPO 计算的最优路径的结果

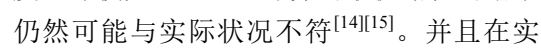
际逃生过程中, 不断在线计算的疏散路径 会发生绕远的情况, 延长疏散时间, 同 时, 不断重复的在线路径优化过程会加重 疏散人员的心里压力, 延缓行动力, 不利 于高层火灾的有效疏散。有效的疏散策略 是用最小疏散时间实现最大的人员疏散数 [16]。因此为了解决这些问题, 本文探究了 CEPO(Co-evolutionary path optimization) 方 法的可行性, 对 DPO 方法进行高层火灾疏 散效率评估。结果表明 CEPO 方法可将离 线路径优化过程和动态环境变化协同进 化, 充分利用现有的火灾动态预测模型

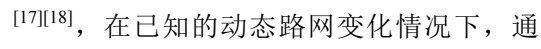
过一次离线优化解决 DPO 方法存在的不 足, 有效提高高层建筑火灾疏散的效率。

\section{2. 最优疏散路径的实现}

\subsection{DPO 方法和 CEPO 方法}

传统的 DPO 方法是结合路网变化实时 的不断的在线重计算。每次路网发生变化 时, DPO 就需要重新规划路径。由于 DPO 方法在路径搜索过程中不能同时考虑给定 的动态路网变化, 所以每次规划的路径只 能满足当前状态的最优, 因而规划出来的 最优路径会与实际情况不符。主要原因是 DPO 忽略了动态路网变化的可预测性, 无 法将动态路径优化过程与实际路网变化过 程协同起来。那么能否通过改进 DPO 方法 实现协同进化路径优化(CEPO), 即最优路 径的搜索过程可以和动态路网变化过程同 步进行。

设路网 $G=(V, E), V, E$ 分别是结点和 链接的集合。假设一个网络有 $\mathrm{N}_{\mathrm{N}}$ 个结点, $N_{E}$ 条链接, 则路径网络可用 $N_{N} * N_{N}$ 的邻接 矩阵 $\mathrm{R}$ 表示。通过参考文献[19]中对 DPO 方法和 CEPO 方法的数学描述与分析可 知, 在路径搜索过程中, DPO 关心结点间 是否有链接, 是基于链接分析的计算操 作。而 CEPO 关心的是哪些时刻链接是可 通行的, 是基于仿真时间长度单位的基本 计算分析操作, 分析各个预测时刻的仿真 时间单位内的个体行为。由于火灾的动态 变化也是基于仿真时间长度的变化, 因此 可以很好地和基于时间单位长度分析操作 的算法完美结合。但现存的 DPO 方法只能 够进行基于链接的计算分析操作, 而把这 种操作改为基于仿真时间长度的操作是非 常困难的, 因为每条路径跨越的仿真时间 单位长度是不确定的。所以将基于时间长 度变化的火灾与基于链接分析的算法结合 来实现协同进化路径优化 (CEPO) 是很难 的, 故两者不能兼容。

\section{CEPO 方法的实现过程}

为了解决 CEPO 问题, 我们需要有基 于单位时间长度进行分析操作的算法, 幸 运的是, 涟渏扩散算法 (Ripple Spreading Algorithm, RSA) 就是基于仿真时间长度分 析的算法。 


\section{1 涟渏扩散算法的基本理念}

涟渏扩散算法受启发于自然界的涟漪 扩散现象, 揭示了一种优化原则, 即, 涟 渏在水面上以相同速度向四周扩散, 总会 率先到达离涟渏中心最近的点, 到空间中 各处的时间是由该处到涟渏中心的距离决 定的 ${ }^{[20]}$ 。在路网中, 涟渏扩散算法的搜索 过程就像在路网结点间进行的涟渏接力 赛。设置初始激励涟渏, 即路径搜索的起 点, 当初始涟渏到达其他未被激活过的结 点后, 新的结点就会被激活产生涟渏, 与 该结点链接的所有结点都被激活过后, 该 结点就停止扩散而消亡。当目的结点第一 次被某个涟渏到达时, 第一最短路径已经 找到, 整个涟渏接力赛结束。通过 RSA 的 搜索过程可以看出, RSA 是分散式的、基 于多智能体的、自下而上的仿真模型 ${ }^{[20]}$, 而非集中式的、自上而下的逻辑算法。在 RSA 中, 只需要设置好激励涟渏和涟渏扩 散行为, 模拟涟渏扩散过程, 当涟渏扩散 结束后, 最终结果自然就是最优路径, 并 不需要再进行全局搜索, 计算从起点到中 间结点的距离并进行排序, 这使得多智能 体分散式仿真模型 RSA 具有更高的修改灵 活性, 方便我们计算各种路径优化问题。 文献[21][22]就是基于这种优化原则创新性 的提出了涟渏扩散算法 RSA 用以求解多种 最短路径问题。接下来将阐释如何改进 RSA 来实现高层建筑火灾下的协同进化路 径优化。

\section{2 应用 RSA 实现高层建筑火灾情况下 的 CEPO}

由于 DPO 方法是基于链接分析操作的 算法, 在单次优化过程中只能把路径优化 问题做静态路径规划问题处理, 无法将基 于单位仿真时间变化的火灾动态有效协 同。RSA 算法恰好是基于单位仿真时间内 的模拟操作, 涟渏激活、扩散行为都是基 于单位仿真时间内的变化, 这就使得 RSA 扩散算法为解决 CEPO 问题提供了可能。 在每个单位仿真时间内, 涟渏的扩散、结 点间涟渏激励过程就可以顺利地与火灾对 路网结点造成的影响相结合。也就是说,
在每个单位仿真时间内, CEPO 一方面要利 用现有的火灾动力学模型获取各个时刻火 灾动态变化的参数, 更新由它造成的路网 变化情况; 另一方面还要同步模拟对应的 单位仿真时间内的涟渏扩散和激活操作。

为了解决 CEPO, 需要对 RSA 做进一 步的改进。通过对 CEPO 的描述, 我们首 先需要获取火灾在各个时刻对路网造成的 变化, 同时将其更新到 RSA 的涟渏接力赛 中 $^{[20]}$ 。其次不同个体的物理属性和链接等 级设定响应链接上的涟渏扩散速度。例如 在本文高层建筑中, 被疏散人员攀爬楼梯 的速度和在楼层内行走的速度是存在差异 的; 不同性别、不同年龄的被疏散人的身 体物理条件也不尽相同, 所以被疏散人员 的逃生速度也就不同。因此在涟渏扩散过 程中, 结点上的涟渏扩散速度由逃生人员 的物理特性和链接等级共同决定, 结点自 主判断链接上的涟渏扩散速度。最后, 设 置逃生人员不存在不可通行区等待的行 为。火灾发生后, 一般对人员造成致命伤 害的主要原因分为火焰直接对被疏散人员 的身体伤害, 和火焰在蕞延过程中燃烧产 生的有毒气体, 例如 $\mathrm{CO}$, 被疏散人员吸入 后会造成急性中毒, 表现为意识不清, 行 走迟缓, 呼吸变慢等反应。所以, 在高层 火灾情况下, 被疏散人员不存在不可通行 区等待的行为, 这一点有别于文献[19]中提 出的台风情景下的应急方法。台风情境 下, 出行人员可进行短暂的等待行为, 待 台风过境后再继续行驶, 从而保证出行人 的安全。所以在文献[18]中引入了结点的等 待行为, 但在火灾情况下, 这种结点上的 等待行为是非常不可取的。

\section{4. 仿真实验}

\section{1 建筑结构模型}

为了评估上述 DPO 方法和 CEPO 方法 在高层建筑火灾中的疏散效果, 在此, 我 们以北京某一高层办公大楼为例构建建筑 模型, 进行初步仿真实验。实体建筑最长 $45 \mathrm{~m}$, 最宽 $27 \mathrm{~m}$, 每楼层高 $3 \mathrm{~m}$, 占地 $1145 \mathrm{~m}^{2}$, 共 12 层高, 无地下室。在整个建 筑的底层设有两个安全出口, 分别位于该 
高层建筑一楼的南侧和东侧, 大楼一楼平 面图如图 1, 其余楼层结构如图 2 图 3 大楼 内部设有两部电梯, 火灾发生后停止使 用。在本文的仿真实验中, 我们对建筑的 长宽进行一定比例的放大, 仿真建筑最长 $36 \mathrm{~m}$, 最宽 $96 \mathrm{~m}$, 每层结构没有发生变化, 目的是将原本只能容纳 600 人的办公大厦扩 大到可容纳 1147 人。

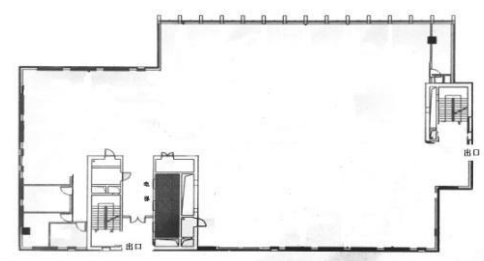

图 1. 北京市某高层建筑一层平面图

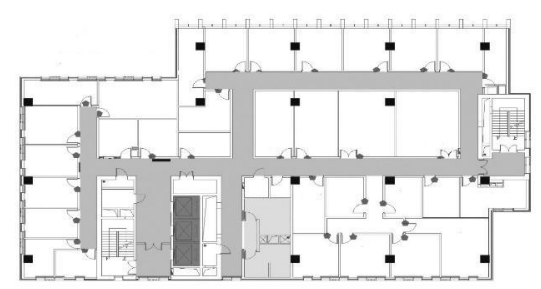

$\square$ 楼道 $\square$ 电梯

图 2. 北京市某高层建筑奇数楼层平面图

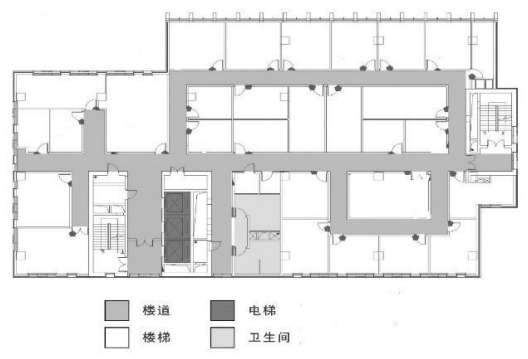

图 3. 北京市某高层建筑偶数楼层平面图

\section{2 疏散者速度的确定}

疏散者由于其年龄、性别的生理特征 不同, 造成个体物理属性存在差异, 例 如: 男性疏散者的行走速度、身体运动
性、灵活性一般都高于女性疏散者 ${ }^{[23]}$ 。因 此, 本文参考了基于以 buildingEXODUS 的 POPULATION 模式, 结合文献[23], 根据 文献[24]提供的中国人年龄、身高和体重关 系的统计数据, 建立中国人生理特征的物 理属性均值表, 如表 1 所示。

疏散人员的物理属性一般包括性别比 例情况, 年龄分布等, 这些参数都是影响 疏散过程的重要参数。本文研究的高层建 筑模型是办公大厦, 建筑楼内办公人员基 本固定, 因此可以根据相应比例设置默认 疏散者的平均行走速度、反映时间等。依 据年龄和性别进行分类。本楼 30 岁左右年 轻人最多, 其次是 40-50 岁年龄的人群, 因 此假设本楼人员的年龄分布在 [20-50]之 间, 该建筑内的女性人数与男性人数比为 1: 1, 女性年龄和男性年龄在 [20-50] 上均 匀分布。根据表 1 按人员性别比例, 年龄分 布进行平均计算, 默认个体的物理属性值 如表 2 。由于每楼层人员密度不大, 可以满 足快速行走的条件, 因此我们主要考虑火 灾情况下人员的快速行走速度和楼梯的攀 爬速度。实验中设定该高层建筑共有办公 人员 1147 人, 平均分配在各楼层, 平均每 $2.76 \mathrm{~m} 2 /$ 人。

\begin{tabular}{cc}
\multicolumn{2}{c}{ 表 1. 默认个人物理属性值 } \\
\hline 属性 & 默认值 \\
\hline 年龄 & 35 岁 \\
身高 & $1.65 \mathrm{~m}$ \\
体重 & $56.2 \mathrm{~kg}$ \\
反应时间 & $0.0 \mathrm{~s}$ \\
快速行走时间 & $1.29 \mathrm{~m} / \mathrm{s}$ \\
爬行速度 & $0.26 \mathrm{~m} / \mathrm{s}$ \\
\hline
\end{tabular}

\section{3 火灾场景设计}

借助火灾动力学软件 (Fire Dynamics Simulator, FDS), 结合该高层建筑的结 构, 设置发生火灾的危险情景。火灾中的 $\mathrm{CO}$ 浓度、 $\mathrm{CO} 2$ 浓度和温度的扩散速度是影 响疏散人安全的重要参数。本文参考文献 [25]中的火灾扩散行为, 获取各个时刻的火 灾扩散参数。 
表 2. 基于中国人生理特征设定的个体物理属性均值

\begin{tabular}{cccccclllll}
\hline $\begin{array}{c}\text { 性 } \\
\text { 别 }\end{array}$ & 年龄 & $\begin{array}{c}\text { 身高 } \\
(\mathrm{cm})\end{array}$ & $\begin{array}{c}\text { 体重 } \\
(\mathrm{kg})\end{array}$ & $\begin{array}{l}\text { 运动 } \\
\text { 性 }\end{array}$ & $\begin{array}{l}\text { 灵敏 } \\
\text { 度 }\end{array}$ & $\begin{array}{l}\text { 快速行 } \\
\text { 走速度 } \\
(\mathrm{m} / \mathrm{s})\end{array}$ & $\begin{array}{l}\text { 行走速度 } \\
(\mathrm{m} / \mathrm{s})\end{array}$ & $\begin{array}{l}\text { 跳跃速度 } \\
(\mathrm{m} / \mathrm{s})\end{array}$ & $\begin{array}{c}\text { 爬行速度 } \\
(\mathrm{m} / \mathrm{s})\end{array}$ \\
\hline 男 & $20 \sim 30$ & 1.7 & 60.00 & 1.0 & 5 & 1.5 & 1.35 & 1.20 & 0.3 \\
女 & $20 \sim 30$ & 1.6 & 52.00 & 0.9 & 4.5 & 1.35 & 1.215 & 1.08 & 0.27 \\
男 & $30 \sim 40$ & 1.7 & 60.00 & 0.9 & 4.5 & 1.35 & 1.22 & 1.08 & 0.27 \\
女 & $30 \sim 40$ & 1.6 & 52.00 & 0.81 & 4.05 & 1.22 & 1.10 & 1.00 & 0.24 \\
男 & $40 \sim 50$ & 1.69 & 60.50 & 0.81 & 4.05 & 1.22 & 1.10 & 1.00 & 0.24 \\
女 & $40 \sim 50$ & 1.59 & 52.50 & 0.73 & 3.65 & 1.10 & 1.00 & 0.88 & 0.22 \\
男 & $50 \sim 60$ & 1.68 & 61.00 & 0.73 & 3.65 & 1.10 & 1.00 & 0.88 & 0.22 \\
女 & $50 \sim 60$ & 1.58 & 53.00 & 0.68 & 3.28 & 1.00 & 0.90 & 0.80 & 0.20 \\
\hline
\end{tabular}

文献[25]对要模拟的区域建立了数值模 型, 设置火点位置, 在火点位置沿 $\mathrm{X}$ 轴、 $\mathrm{Y}$ 轴方向 $10 \mathrm{~m}$ 处设置四个气体监测设备, 对 温度、 $\mathrm{CO}$ 浓度、 $\mathrm{CO} 2$ 浓度进行监测。由表 3 可见, $\mathrm{CO}$ 气体和温度在 $10 \mathrm{~m}$ 处到达临界 值的时间分别是 $72.69 \mathrm{~s}$ 和 $245.32 \mathrm{~s}$ 。需要说 明的是危险临界值不一定对被疏散人员造 成生命威胁, 但是超过该临界值就会对被 疏散人员产生致死威胁。同时临界值对不 同物理生理特征的人造成的影响不同, 不 同年龄、性别等承受 $\mathrm{CO}$ 浓度、温度的极限 也不同, 因此表 3 主要参考文献[26]-[29]确 定平均危险临界值。

\begin{tabular}{|c|c|c|c|}
\hline $\begin{array}{l}\text { 影响 } \\
\text { 因素 }\end{array}$ & $\begin{array}{l}\text { 危险临界 } \\
\text { 值 }\end{array}$ & $\begin{array}{l}\text { 最早到达 } \\
\text { 时间 }\end{array}$ & $\begin{array}{l}\text { 扩散速 } \\
\text { 度 }\end{array}$ \\
\hline $\begin{array}{l}\mathrm{CO} \text { 浓 } \\
\text { 度 }\end{array}$ & $2.3 \mathrm{~g} / \mathrm{m} 3$ & $72.69 \mathrm{~s}$ & $0.137 \mathrm{~m} / \mathrm{s}$ \\
\hline $\begin{array}{l}\mathrm{CO} 2 \\
\text { 浓度 }\end{array}$ & $>6 \% \mathrm{~g} / \mathrm{m} 3$ & - & - \\
\hline 温度 & $120^{\circ}$ & $245.32 \mathrm{~s}$ & 0.041 \\
\hline
\end{tabular}

\section{4 仿真实验}

在本文的单次路径疏散仿真实验中, 我们随机选取该高层建筑中的某一疏散人 员, 结合上述的火灾扩散行为, 进行火灾 下的疏散仿真实验。在本次仿真实验中, 随机选取火灾源位置为七层右侧电梯旁, 坐标 $(96,24,18)$ 。如图 4 和图 5 所示, 红色 圆形区域为高层建筑中火灾源的发生地, 红色方框为该疏散人员的起始地, 蓝色圆 形为最终的安全出口, 从图 4 中可以看出, 起初 DPO 是想通过右侧的楼梯间逃生, 但 当疏散人员走到顶层的楼梯口时, 火灾已
经蔓延到下层楼梯间, 原有路径规划无法 执行, 经过实时重计算, DPO 从下一楼层 走廊, 绕道走左侧楼梯间顺利逃生。而采 用 CEPO 方法则可以直接避免在线重计 算, 如图 5 所示, CEPO 通过一次离线路径 规划就可获得实际最优路径, 不仅减短了 疏散路径长度, 也尽可能的减少疏散时 间, 具体仿真结果见表 4 。

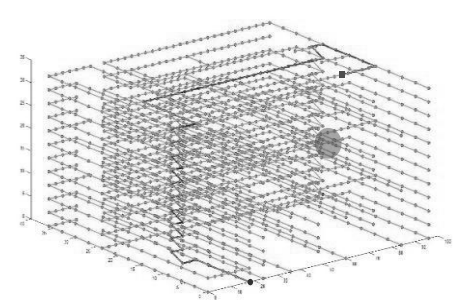

图 4. DPO 方法模拟结果

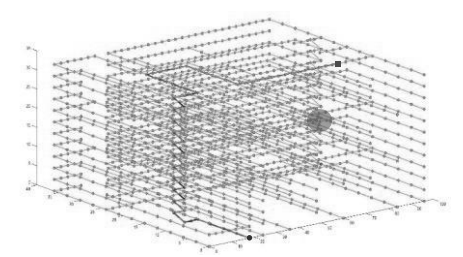

图 5. 基于 RSA 的 CEPO 模拟结果

表 4. DPO 方法和 CEPO 方法单次疏散仿真结果

\begin{tabular}{l|l|l}
\hline & DPO 方法 & CEPO 方法 \\
\hline 逃生时间 $/ \mathrm{s}$ & 355 & 330 \\
\hline 逃生路径 $/ \mathrm{m}$ & 205.99 & 175.14 \\
\hline 计算时间 $/ \mathrm{s}$ & 3.95 & 5.04 \\
\hline
\end{tabular}

为了能够更加全面的评估 DPO 方法和 
CEPO 方法的优劣, 又对整个楼层的所有人 员进行了仿真疏散。此次仿真实验的火灾 源发生地为七楼中部的配电室, 坐标 $(66,24$, 18), 采用该两种方法同时疏散分布在各楼 层内的所有人员, 得仿真结果如表 5 所示。

表 5. CEPO 和 DPO 两种方法疏散所有人员的仿

\begin{tabular}{l|l|l}
\multicolumn{3}{|c}{ 真结果 } \\
\hline & DPO 方法 & CEPO 方法 \\
\hline $\begin{array}{l}\text { 平均每人逃生时 } \\
\text { 间 } / \mathrm{s}\end{array}$ & 206 & 202 \\
\hline 未逃生人数/人 & 16 & 1 \\
\hline
\end{tabular}

\section{5 结果分析}

通过案例实验, 对个体和整个楼层人 员的疏散过程进行评估。传统的动态路径 优化算法 DPO 在火灾疏散效率方面不及 CEPO 方法。虽然 DPO 方法也考虑了火灾 动态扩散过程, 但每一次的疏散路径只能 满足当前时刻最优。根据仿真实验可知, DPO 不断在线的实时规划只会增加疏散人 员逃跑的路径长度, 延长逃生时间。如果 逃生路线总是处于变化中, 无效的逃生规 划会加重疏散人的不安和恐慌心理, 反而 不利于火灾紧急情况下的有效疏散。

比较两种算法, 新方法 CEPO 可以通 过一次离散路径优化实现最短路径, 由于 默认每位逃生人员物理属性一致, 比较平 均逃生时间也可以评估所有人员疏散路径 的长度, 显然在火灾疏散效率方面, CEPO 方法更具有优势：

1、关于疏散效率。通过比较单位时间 内两种方法成功疏散的人数, 表 5 可知, CEPO 方法的成功疏散率比 DPO 方法高出 $4.7 \%$ 。

2、在高层火灾的危险态势下, DPO 方 法的在线重计算, 不仅增加了计算机的计 算时间, 而且在规划路径可能绕远的情况 下延长疏散时间, 不利于在安全时间内帮 助人员逃生, 反观 CEPO 方法, 不仅可以 缓解在线重计算对疏散者造成的心里压 力, 还可以大大降低计算时间, 提高疏散 效率。

\section{5. 结论}

本文主要在高层建筑火灾情景下探索 了目前 DPO 方法在高层建筑疏散模型中应 用的弊端, 同时对传统 DPO 方法进行了疏 散效率的评估。针对 DPO 方法的不足, 探 究了协同进化路径优化(CEPO)和涟渏扩散 算法(RSA)在火灾情景下的应用。由于基于 单位链接搜索操作的 DPO 方法无法同步基 于单位时间变化的火灾, 造成 DPO 无法考 虑火灾对路网造成的未来变化, 规划的路 径无法满足实际理论最优路径。与现有的 动态路径优化(DPO)方法不同, CEPO 不需 要在线实时优化, 通过可以预测和模拟的 火灾扩散行为, CEPO 在一次性的离线优化 过程中的会同时考虑此时路网的变化, 所 以, 离线优化的结果就能保证实际疏散路 径的最优性, 极大的减少在线计算次数。 通过北京某一高层办公楼为例, 我们进行 了仿真实验, 实验结果清晰的表明了 CEPO 方法提高了高层建筑的火灾疏散效果, 减 少不断在线重新规划路径情况下, 疏散人 员由于不安和恐慌误入火灾危险区的风 险。今后将从风险角度更多的探索影响疏 散风险的规律, 探索不同灾害情景下的不 同路网环境的抗打击能力, 提高基于 RSA 的 CEPO 方法的应用广度, 降低不同路网 系统在灾害下的脆弱性。

\section{参考文献}

[1] 黄莺. 公共建筑火灾风险评估及安全管 理方法研究, 西安: 西安建筑科技大 学, 2009.

[2] 侣记清, 齐鸿涁. 高层民用建筑设计的 几个消防问题, 中山大学学报论丛, 2 1(5):147-149, 2001.

[3] M. Goodwin, OC. Granmo, J Radianti, Escape planning in realistic fire scenarios with Ant Colony Optimisation, Applied Intelligence, 42(1): 24-35, 2015.

[4] 吕春杉, 翁文国, 杨锐. 基于运动模式 和元胞自动机的火灾环境下人员疏散 模型, 清华大学学报 (自然科学版), 47(12), 2007. 
[5] C.M. Zhao, S M. Lo, J A. Lu, et al, A simulation approach for ranking of fire safety attributes of existing buildings, Fire Safety Journal, 39(7): 557-579, 2004

[6] S.M. Lo, H C. Huang, P. Wang, et al, A game theory based exit selection model for evacuation, Fire Safety Journal, (7): 364-369, 2006.

[7] 刘勇.基于蚁群算法的应急救援最优路 径研究, 北京: 中国地质大学, 2010 .

[8] 宋洋, 徐桢, 王燕青. 基于蚂蚁算法的 危险品运输路径优化研究, 安全与环 境工程, (1): 148-152, 2014.

[9] 段鹏飞, 熊盛武, 李辉, 面向大型场 馆疏散的改进多蚁群算法, 计算机应 用研究, 30(2): 357-363, 2013.

[10] G. Ramalingam and T. Reps, On the computational complexity of dynamic graph problems, Theoretical Computer Science, 158:233-277, 1996.

[11] D. Frigioni, A. Marchetti-Spaccamela and U. Nanni, Fully dynamic algorithms for maintaining shortest paths trees. Journal of Algorithms, 34:251-281, 2000.

[12] D. Frigioni, A. Marchetti-Spaccamela and U. Nanni, Semidynamic algorithms for maintaining single-source shortest path trees. Algorithm-mica, 22:250-274, 1998.

[13] P. Narvaez, KY. Siu and HY. Tzeng, New dynamic algorithms for shortest path tree computation, IEEE/ACM Transactions on Networking, 8:734-746, 2000.

[14] 杜鹏桢, 唐振民, 孙研. 一种面向对象 的多角色蚁群算法及其 TSP 问题求解, 控制与决策, 29(10):1729-1736, 2014.

[15] J, Wang, H F. Zhao, S. Winter, Integrating sensing, routing and timing for indoor evacuation. Fire Safety Journal, 78: 111121, 2015.

[16] Lakshay, A. Agarwal, N. Bolia, Route Guidance Map for Emergency Evacuation, Journal of Risk Analysis and Crisis Response,6(3): 135-144, 2016.
[17] 黄维章, 张锁春, 雷光耀. 城市火灾蔓 延的数学模型和计算机模拟, 计算物 理, 10(1), 1993.

[18] 马金金, 黄全义, 刘全义. 基于物联网的 建筑火灾动态监测方法, 清华大学学 报 (自然科学版), 52(11), 2010.

[19] M.K. Zhang, X.B. Hu, Path Optimization Method in Dynamic Weathers, Journal of Risk Analysis and Crisis Response, 6(4):197-205, 2016.

[20] X.B. Hu, M. Wang, M.S. Leeson, E.L. Hines, and E. Di Paolo, A Deterministic Agent-Based Path Optimization Method by Mimicking the Spreading of Ripples, Evolutionary Computation, 24:319-346, 2016.

[21] X.B. Hu, M. Wang, Hu. D, M.S. Leeson, E.L. Hines, and E. Di Paolo, A RippleSpreading Algorithm for the $\mathrm{k}$ Shortest Paths Problem. 2012 the 3rd Global Congress on Intelligent Systems (GCIS 2012), Wuhan, China, 202-208, 2012.

[22] X.B. Hu, Q. Sun, M. Wang, M.S. Leeson, and E.D. Paolo, A Ripple Spreading Algorithm to Calculate the $k$ Best Solutions to the Project Time Management Problem. 2013 IEEE Symposium Series on Computational Intelligence (IEEE SSCI 2013), 16-19 April 2013, Singapore.

[23] M.R. Virkler, Prediction and measurement of travel time along pedestrian routes, Transportation Research Record, 36(16): 37-42m, 1998.

[24] J.X. Wang, B X. Li, The reference value of the height and the weight of Chinese people. Radiation Protection of Medical, 13(3): 3-7, 1993.

[25] 刘毅, 沈斐敏. 考虑灾害实时扩散的室 内火灾疏散路径选择模型. 控制与决策, 2017.

[26] 童庆杰, 权高蜂, 邵力. 火灾事故中人 的心里及行为分析, 合肥工业大学学报 (社会科学版), 18(3): 159-162, 2004. 
[27] 邱榕, 范维澄. 火灾常见有害燃烧产物 的生物毒理 (I) 一氧化碳、氭化氢, 火灾科学, 10(3):154-158, 2001.

[28] 王新民, 姚建, 彭欣. 火灾时期致命因 素危害时间的研究, 消防科学与技 术, 24(1):28-30, 2005.

[29] P.J. DiNenno, D. Drysdale, C.L. Beyler, et al. The SFPE handbook of fire protection engineering, 3rd edition. Massachusetts: Society of Fire Protection Engineers/National Fire Protection Association, 2002. 